\title{
An enChIP system for the analysis of bacterial genome functions
}

\author{
Toshitsugu Fujita ${ }^{1,2}$, Miyuki Yuno ${ }^{2}$ and Hodaka Fujii ${ }^{1} 2^{*}$ (i)
}

\begin{abstract}
Objective: The engineered DNA-binding molecule-mediated chromatin immunoprecipitation (enChIP) technology enables purification of specific genomic regions interacting with their associated molecules. In enChIP, the locus to be purified is first tagged with engineered DNA-binding molecules. An example of such engineered DNA-binding molecules to tag the locus of interest is the clustered regularly interspaced short palindromic repeats (CRISPR) system, consisting of a catalytically-inactive form of Cas9 (dCas9) and guide RNA (gRNA). Subsequently, the tagged locus is subjected to affinity purification for identification of interacting molecules. In our previous studies, we developed enChIP systems for analysis of mammalian genome functions. Here, we developed an enChIP system to analyze bacterial genome functions.

Results: We generated a plasmid inducibly expressing Streptococcus pyogenes dCas9 fused to a 3xFLAG-tag (3xFLAGdCas9) in bacteria. Inducible expression of 3xFLAG-dCas9 in Escherichia coli was confirmed by immunoblot analysis. We were able to purify specific genomic regions of E. coli preserving their molecular interactions. The system is potentially useful for analysis of interactions between specific genomic regions and their associated molecules in bacterial cells to understand genome functions such as transcription, DNA repair, and DNA recombination.
\end{abstract}

Keywords: enChIP, dCas9, ChIP, Chromatin immunoprecipitation, CRISPR, Bacteria

\section{Introduction}

To understand the regulatory mechanisms underlying genome functions such as transcription, it is essential to identify the molecules associated with a genomic region of interest in vivo. The engineered DNA-binding molecule-mediated chromatin immunoprecipitation (enChIP) technology we developed recently, enables specific isolation of genomic regions of interest interacting with their associated molecules [1,2]. Examples of such engineered DNA-binding molecules to tag the locus of interest are transcription activator-like proteins [3] and the clustered regularly interspaced short palindromic repeats (CRISPR) system [4, 5] consisting of a catalytically-inactive form of Cas9 (dCas9) and guide RNA (gRNA). Subsequently, the tagged locus is affinity-purified to identify

\footnotetext{
*Correspondence: hodaka@hirosaki-u.ac.jp

${ }^{1}$ Department of Biochemistry and Genome Biology, Hirosaki University Graduate School of Medicine, 5 Zaifu-cho, Hirosaki, Aomori 036-8562, Japan

Full list of author information is available at the end of the article
}

interacting molecules. A locus of interest can be tagged in the cell by expressing engineered DNA-binding molecules $[1,2,6-9]$ (in-cell enChIP) or in vitro using recombinant or synthetic engineered DNA-binding molecules $[10,11]$ (in vitro enChIP). After purification of the locus of interest, mass spectrometry (MS), RNA sequencing, and next-generation sequencing (NGS) can be used to identify proteins $[1,2,6]$, RNAs [7], and genomic regions $[9,11]$ binding to the locus in a non-biased manner.

Previously, we developed enChIP systems for analysis of mammalian genome functions. Here, we report development of an in-cell enChIP system for bacterial cells (Fig. 1a). The system consists of plasmids expressing Streptococcus pyogenes dCas9 fused to a 3xFLAG-tag (3xFLAG-dCas9) and gRNA in bacteria. The developed enChIP system isolated target genomic regions from Escherichia coli. The system might enable identification of molecules associated with a specific genomic region in bacteria, and thus help to elucidate their genome functions.

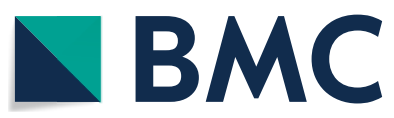

C The Author(s) 2018. This article is distributed under the terms of the Creative Commons Attribution 4.0 International License (http://creativecommons.org/licenses/by/4.0/), which permits unrestricted use, distribution, and reproduction in any medium, provided you give appropriate credit to the original author(s) and the source, provide a link to the Creative Commons license, and indicate if changes were made. The Creative Commons Public Domain Dedication waiver (http://creativecommons.org/ publicdomain/zero/1.0/) applies to the data made available in this article, unless otherwise stated. 


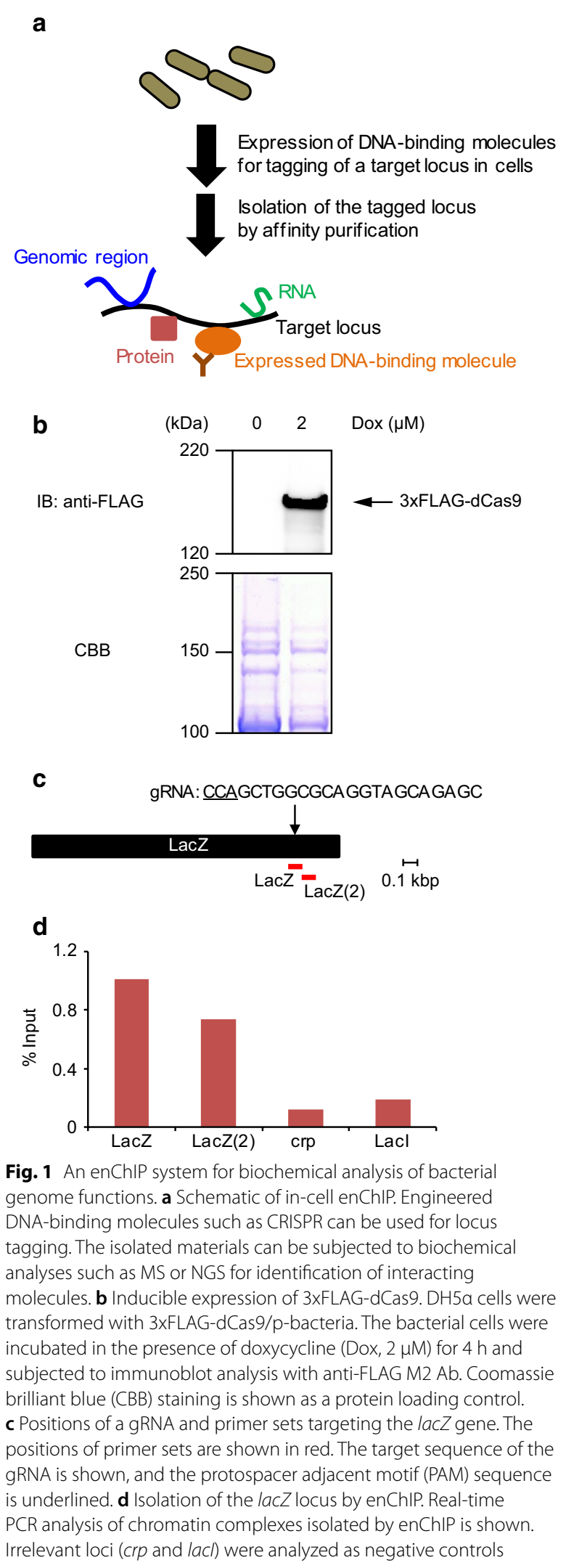

\section{Main text}

Materials and methods Plasmids

To construct the doxycycline (Dox)-inducible 3xFLAGdCas9 expression plasmid 3xFLAG-dCas9/p-bacteria (Addgene \#64325), pdCas9-bacteria (Addgene \#44249) [12] was digested with BglII and BstZ17I and treated with bacterial alkaline phosphatase (E. coli C75) (2120A, Takara-Bio). The double-strand DNA (dsDNA) containing the coding sequence of the 3xFLAG-tag and the $\mathrm{N}$-terminal portion of dCas9 (agatctaaagaggagaaaggatctATGGACTACAAAGACCATGACGGTGATTATAAA GATCATGACATCGATTACAAGGATGACGATGAC AAGCTCATGGATAAGAAATACTCAATAGGCTTA GCTATCGGCACAAATAGCGTCGGATGGGCGGTG ATCACTGATGAATATAAGGTTCCGTCTAAAAAG TTCAAGGTTCTGGGAAATACAGACCGCCACAGT ATCAAAAAAAATCTTATAGGGGCTCTTTTATTT GACAGTGGAGAGACAGCGGAAGCGACTCGTCTC AAACGGACAGCTCGTAGAAGGTATAC) (underlined: 3xFLAG-tag; italic: dCas9) was synthesized (Invitrogen) and digested with BglII and BstZ17I. The cleaved pdCas9bacteria and the synthetic DNA fragment were purified by agarose gel electrophoresis and ligated.

To construct vectors for expression of gRNAs, pgRNAbacteria (Addgene \#44251) [12] was digested with SpeI and HindIII and treated with bacterial alkaline phosphatase (E. coli C75). The dsDNA for targeting the lacZ gene (ACTAGTGCTCTGCTACCTGCGCCAGCGTT TTAGAGCTAGAAATAGCAAGTTAAAATAAGGCT AGTCCGTTATCAACTTGAAAAAGTGGCACCGAG TCGGTGCTTTTTTTGAAGCTT) (underlined: the target sequences) was synthesized (Invitrogen) and digested with SpeI and HindIII. The cleaved pgRNA-bacteria and the synthetic DNA fragment were purified by agarose gel electrophoresis and ligated. For construction of gRNAs targeting the promoter region of the rpoH gene, oligodeoxyribonucleotides (Table 1) were synthesized, annealed, and phosphorylated. Combination of oligodeoxyribonucleotides was; $E$. coli gRNA common: $27816+27817$; rpoH p 158-180: 27818+27819; rpoH p 184-206: $27820+27821$. The annealed dsDNAs were subjected to ligation reactions with the SpeI and HindIII-digested pgRNA-bacteria plasmid.

\section{E. coli strains}

DH5 $\alpha$ (9057, Takara-Bio) was transformed with 3xFLAG-dCas9/p-bacteria alone or together with gRNA expression plasmids, and transformed bacteria were selected with chloramphenicol (Cam) $(25 \mu \mathrm{g} /$ $\mathrm{ml}$ ) for 3xFLAG-dCas9/p-bacteria alone or a combination of Cam $(25 \mu \mathrm{g} / \mathrm{ml})$ and ampicillin (Amp) $(50 \mu \mathrm{g} / \mathrm{ml})$ 
Table 1 Oligodeoxyribonucleotides used in this study

\begin{tabular}{|c|c|c|c|}
\hline Number & Name & Sequence $\left(5^{\prime} \rightarrow 3^{\prime}\right)$ & Experiments \\
\hline 27816 & E. coli gRNA common S & $\begin{array}{l}\text { agttaaaataaggctagtccgttatcaacttgaaaaagtggcaccgagtcg- } \\
\text { gtgcttttttga }\end{array}$ & $\begin{array}{l}\text { Construction of plasmids targeting the rpoH gene } \\
\text { promoter }\end{array}$ \\
\hline 27817 & E. coli gRNA common A & $\begin{array}{l}\text { agcttcaaaaaaagcaccgactcggtgccactttttcaagttgataacg- } \\
\text { gactagcct }\end{array}$ & $\begin{array}{l}\text { Construction of plasmids targeting the rpoH gene } \\
\text { promoter }\end{array}$ \\
\hline 27818 & rpoH p 158-180 S & ctagtgttatactctttccctgcaagttttagagctagaaatagca & $\begin{array}{l}\text { Construction of the plasmid targeting the rpoH gene } \\
\text { promoter 158-180 }\end{array}$ \\
\hline 27819 & rpoH p 158-180 A & tatttaacttgctatttctagctctaaaacttgcagggaaagagtataaca & $\begin{array}{l}\text { Construction of the plasmid targeting the rpoH gene } \\
\text { promoter 158-180 }\end{array}$ \\
\hline 27820 & rpoH p 184-206 S & ctagtcggggtctctttccctgctagttttagagctagaaatagca & $\begin{array}{l}\text { Construction of the plasmid targeting the rpoH gene } \\
\text { promoter 184-206 }\end{array}$ \\
\hline 27821 & rpoH p 184-206 A & tattttaacttgctatttctagctctaaaactagcagggaaagagaccccga & $\begin{array}{l}\text { Construction of the plasmid targeting the rpoH gene } \\
\text { promoter 184-206 }\end{array}$ \\
\hline 27792 & LacZ-E. coli-F & gcgattaccgttgatgttgaagt & Real-time PCR in Figs. 1d and 2d (LacZ) \\
\hline 27793 & LacZ-E. coli-R & agtaaggcggtcgggatagtttt & Real-time PCR in Figs. 1d and 2d (LacZ) \\
\hline 27794 & LacZ-E. coli-F2 & aaaactatcccgaccgccttact & Real-time PCR in Fig. 1d (LacZ(2)) \\
\hline 27795 & LacZ-E. coli-R2 & gggaagacgtacggggtatacat & Real-time PCR in Fig. 1d (LacZ(2)) \\
\hline 27796 & crp-E. coli-F & tcacttcagagaaagtgggcaac & Real-time PCR in Fig. 1d (crp) \\
\hline 27797 & crp-E. coli-R & gtcatagcgtctggttgttttgc & Real-time PCR in Fig. 1d (crp) \\
\hline 27798 & Lacl-E. coli-F & cgtcagtgggctgatcattaact & Real-time PCR in Fig. 1d (Lacl) \\
\hline 27799 & Lacl-E. coli-R & atcaagaaataacgccggaacat & Real-time PCR in Fig. 1d (Lacl) \\
\hline 27902 & rpoH-E. coli-F & aagcttgcattgaacttgtggat & Real-time PCR in Fig. 2d (rpoH-prom) \\
\hline 27903 & rpoH-E. coli-R & tatcttctggcgcttcagtggta & Real-time PCR in Fig. 2d (rpoH-prom) \\
\hline 27896 & rpoH-coding-F & tacgttctgcgtaactggcgtat & Real-time PCR in Fig. 2d (rpoH-cod) \\
\hline 27897 & rpoH-coding-R & accatttcgacttcatcctggtt & Real-time PCR in Fig. 2d (rpoH-cod) \\
\hline
\end{tabular}

for 3xFLAG-dCas9/p-bacteria plus gRNA expression plasmid.

\section{Confirmation of inducible expression of 3xFLAG-dCas 9}

DH5 $\alpha$ transformed with 3xFLAG-dCas9/p-bacteria was cultured in $2 \mathrm{ml}$ of LB media containing Cam $(25 \mu \mathrm{g} / \mathrm{ml})$ at $37^{\circ} \mathrm{C}$ overnight with shaking. One hundred microliters of the culture liquid was mixed with $900 \mu \mathrm{l}$ of LB media containing Cam $(25 \mu \mathrm{g} / \mathrm{ml})$ and incubated for $1 \mathrm{~h}$ with shaking. Subsequently, Dox $(2 \mu \mathrm{M})$ was added to the culture media for induction of expression of 3xFLAG-dCas9. After incubation with shaking for $4 \mathrm{~h}, 400 \mu \mathrm{l}$ of the culture liquid was centrifuged at $5000 \mathrm{rpm}$ for $1 \mathrm{~min}$, and the bacterial pellets were suspended in $100 \mu \mathrm{l}$ of $4 \times$ SDS buffer. After boiling at $100{ }^{\circ} \mathrm{C}$ for $5 \mathrm{~min}, 10 \mu \mathrm{l}$ of the sample was subjected to SDS-PAGE with a $5-20 \%$ gradient gel followed by immunoblot analysis with anti-FLAG M2 antibody (Ab) (F1804, Sigma-Aldrich).

\section{enChIP-real-time PCR}

DH5 $\alpha$ cells transformed with 3xFLAG-dCas9/p-bacteria alone or together with a gRNA expression plasmid were cultured in $5 \mathrm{ml}$ of LB media containing Cam
$(25 \mu \mathrm{g} / \mathrm{ml})(3 x F L A G-d C a s 9 / p-b a c t e r i a)$ or Cam $(25 \mu \mathrm{g} /$ $\mathrm{ml})$ plus Amp $(50 \mu \mathrm{g} / \mathrm{ml})$ (3xFLAG-dCas9/p-bacteria and a gRNA expression plasmid) overnight at $37{ }^{\circ} \mathrm{C}$ with shaking. The bacterial culture was added to $100 \mathrm{ml}$ of LB media containing the same antibiotics and cultured at $37^{\circ} \mathrm{C}$ with shaking. When $\mathrm{OD}_{600}$ of the culture media reached 0.5, Dox $(2 \mu \mathrm{M})$ was added for induction of 3xFLAG-dCas9 expression. After incubation at $37^{\circ} \mathrm{C}$ for $4.5 \mathrm{~h}$ with shaking, the bacterial cells were fixed with $1 \%$ formaldehyde at $37^{\circ} \mathrm{C}$ for $5 \mathrm{~min}$ and neutralized with glycine at room temperature for $10 \mathrm{~min}$. After centrifugation, the cell pellets were suspended in $800 \mu \mathrm{l}$ of modified lysis buffer $3[10 \mathrm{mM}$ Tris, $\mathrm{pH} 8.0,1 \mathrm{mM}$ EDTA, $0.5 \mathrm{mM}$ EGTA, $150 \mathrm{mM} \mathrm{NaCl}, 0.1 \%$ sodium deoxycholate, $0.1 \%$ SDS, and $1 \times$ protease inhibitors (Complete-Mini, EDTA-free, 11836170001, Roche)], and DNA was fragmented by sonication using Ultrasonic disruptor UD-201 (TOMY SEIKO) with conditions: Output, 3; Duty, 100\% (continuous); Time, Free; 6 cycles of sonication for $10 \mathrm{~s}$ and cooling on ice for $20 \mathrm{~s}$ (the average length of chromatin fragments was $1 \mathrm{kbp}$ ). The sonicated chromatin was subjected to enChIP-realtime PCR analysis as described previously $[1,2]$. Primers used in the analysis are shown in Table 1. 


\section{Results and discussion}

\section{An enChIP system for the locus-specific biochemical} analysis of bacterial genome functions

To analyze the locus-specific functions of the bacterial genome biochemically, we constructed a bacterial expression plasmid (3xFLAG-dCas9/p-bacteria (Addgene \#64325)) encoding S. pyogenes dCas9 fused with the 3xFLAG-tag. The E. coli $\mathrm{DH} 5 \alpha$ strain was transformed with 3xFLAG-dCas9/p-bacteria, and inducible expression of 3xFLAG-dCas 9 by Dox was confirmed by immunoblot analysis with anti-FLAG Ab (Fig. 1b).

Next, for enChIP analysis, we designed a gRNA targeting the coding region of the lacZ gene (Fig. 1c). We transformed DH5 $\alpha$ with 3xFLAG-dCas9/p-bacteria together with a gRNA expression plasmid targeting the lac $Z$ gene in the DH5 $\alpha$ genome. Four hours after the addition of Dox $(2 \mu \mathrm{M})$, cells were crosslinked with formaldehyde and subjected to sonication for fragmentation of chromatin DNA. Next, chromatin complexes containing 3xFLAG-dCas9/gRNA were immunoprecipitated with anti-FLAG Ab, and DNA was purified. Real-time PCR showed enrichment of the lacZ gene (Fig. 1c, d). The percentage of input of negative control genomic regions (the crp and lacI genes) was markedly lower than that of the lacZ gene (Fig. 1d). These results showed that the bacterial enChIP system can specifically and efficiently isolate target genomic regions.

\section{enChIP analysis of an endogenous gene promoter}

Next, we targeted the promoter region of an endogenous gene, $r p o H$, which encodes RNA polymerase sigma (32) factor [13]. This gene is a single operon gene and one of the essential genes of E. coli [Profiling of E. coli Chromosome (PEC): https://shigen.lab.nig.ac.jp/ecoli/pec/]. First, we designed two gRNAs targeting the promoter region of the rpoH gene (Fig. 2a). Next, we transformed DH5 $\alpha$ with 3xFLAG-dCas9/p-bacteria alone or together with one of two gRNA expression plasmids targeting the promoter region. After enChIP, real-time PCR showed that the promoter region of the rpoH gene was specifically enriched only in the immunoprecipitants prepared from DH5 $\alpha$ cells transformed with both 3xFLAG-dCas9/p-bacteria and either one of two gRNA expression plasmids, but not from those transformed with 3xFLAG-dCas9/p-bacteria alone (Fig. 2b, c). The percentage of input of negative control genomic regions (the coding region of the rpoH gene or the lac $Z$ gene) was markedly lower than that of the rpoH promoter region (Fig. 2b, c). These results confirmed that the bacterial enChIP system can specifically and efficiently isolate endogenous target genomic regions.

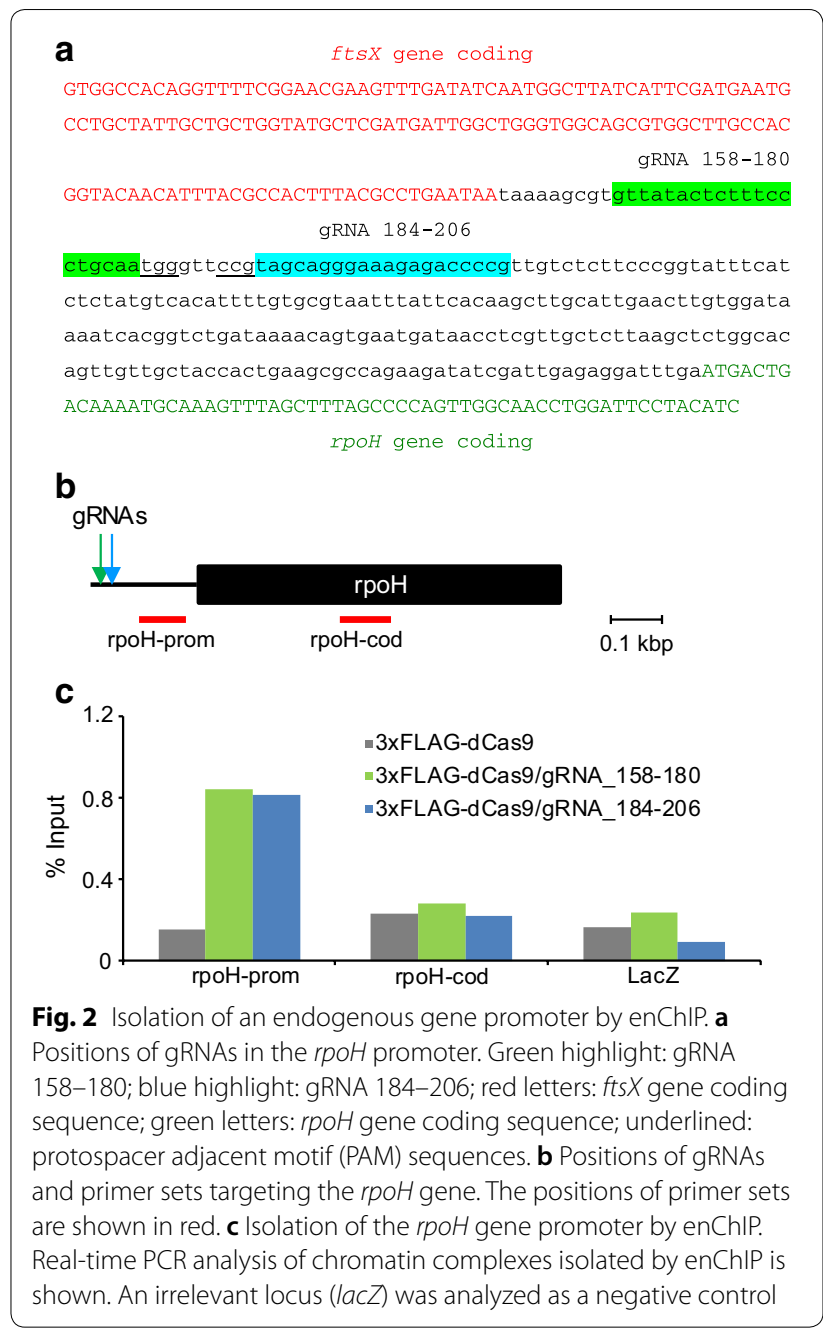

\section{Conclusions}

In this study, we developed an enChIP system for analysis of bacterial genomes. This system enables efficient isolation of specific genomic regions from the $E$. coli genome while preserving their chromatin structures, and potentially contributes to the understanding of bacterial genome functions such as transcription and DNA repair.

\section{Limitations}

Further studies might be necessary to assess the utility of this system combined with MS and NGS to identify molecules associated with the target genomic regions in bacteria.

\section{Abbreviations}

enChIP: engineered DNA-binding molecule-mediated chromatin immunoprecipitation; CRISPR: clustered regularly interspaced short palindromic repeats; dCas9: a catalytically-inactive form of Cas9; gRNA: guide RNA; NLS: nuclear 
localization signal; MS: mass spectrometry; NGS: next-generation sequencing; Cam: chloramphenicol; Amp: ampicillin; Ab: antibody.

\section{Authors' contributions}

HF conceived the idea of development of an enChIP system for analysis of bacterial genome functions and constructed expression plasmids. TF and HF designed and performed experiments and wrote the manuscript. MY performed experiments. HF directed and supervised the study. All authors read and approved the final manuscript.

\section{Author details}

1 Department of Biochemistry and Genome Biology, Hirosaki University Graduate School of Medicine, 5 Zaifu-cho, Hirosaki, Aomori 036-8562, Japan. ${ }^{2}$ Chromatin Biochemistry Research Group, Combined Program on Microbiology and Immunology, Research Institute for Microbial Diseases, Osaka University, 3-1 Yamadaoka, Suita, Osaka 565-0871, Japan.

\section{Acknowledgements}

We thank Stanley Qi for pdCas9-bacteria (Addgene \#44249) and pgRNAbacteria (Addgene \#44251).

\section{Competing interests}

TF. and H.F. have patents on enChIP (Patent name: Method for isolating specific genomic region using molecule binding specifically to endogenous DNA sequence; Patent number: Japan 5,954,808; Patent application number: WO2014/125668). T.F. and H.F. are founders of Epigeneron, Inc.

\section{Availability of data and materials}

All data generated or analyzed during the current study are included in this published article.

\section{Consent for publication}

Not applicable.

\section{Ethics approval and consent to participate} Not applicable.

\section{Funding}

This work was supported by the Takeda Science Foundation (T.F.), Grant-in-Aid for Scientific Research (C) (\#15K06895) (T.F.), and Grant-in-Aid for Scientific Research (B) (\#15H04329) (T.F., H.F.), 'Transcription Cycle' (\#15H01354) (H.F.) from the Ministry of Education, Culture, Sports, Science and Technology of Japan.

\section{Publisher's note}

Springer Nature remains neutral with regard to jurisdictional claims in published maps and institutional affiliations.
Received: 19 April 2018 Accepted: 5 June 2018

Published online: 14 June 2018

\section{References}

1. Fujita T, Fujii H. Efficient isolation of specific genomic regions and identification of associated proteins by engineered DNA-binding moleculemediated chromatin immunoprecipitation (enChIP) using CRISPR. Biochem Biophys Res Commun. 2013;439:132-6.

2. Fujita T, Asano Y, Ohtsuka J, Takada Y, Saito K, Ohki R, Fujii H. Identification of telomere-associated molecules by engineered DNA-binding molecule-mediated chromatin immunoprecipitation (enChIP). Sci Rep. 2013;3:3171.

3. Bogdanove AJ, Voytas DF. TAL effectors: customizable proteins for DNA targeting. Science. 2011;333:1843-6.

4. Harrison MM, Jenkins BV, O'Connor-Giles KM, Wildonger J. A CRISPR view of development. Genes Dev. 2014:28:1859-72.

5. Wright AV, Nuñez JK, Doudna JA. Biology and applications of CRISPR systems: harnessing nature's toolbox for genome engineering. Cell. 2016;164:29-44.

6. Fujita T, Fujii H. Identification of proteins interacting with genomic regions of interest in vivo using engineered DNA-binding moleculemediated chromatin immunoprecipitation (enChIP). Bio Protoc. 2014;4:e1124

7. Fujita T, Yuno M, Okuzaki D, Ohki R, Fujii H. Identification of non-coding RNAs associated with telomeres using a combination of enChIP and RNA sequencing. PLoS ONE. 2015;10:e0123387.

8. Fujita T, Yuno M, Fujii H. Allele-specific locus binding and genome editing by CRISPR at the p16INK4a locus. Sci Rep. 2016;6:30485.

9. Fujita T, Yuno M, Suzuki Y, Sugano S, Fujii H. Identification of physical interactions between genomic regions by enChIP-Seq. Genes Cells. 2017:22:506-20.

10. Fujita T, Yuno M, Fujii H. Efficient sequence-specific isolation of DNA fragments and chromatin by in vitro enChIP technology using recombinant CRISPR ribonucleoproteins. Genes Cells. 2016;21:370-7.

11. Fujita T, Kitaura F, Yuno M, Suzuki Y, Sugano S, Fujii H. Locus-specific ChIP combined with NGS analysis reveals genomic regulatory regions that physically interact with the Pax5 promoter in a chicken B cell line. DNA Res. 2017;24:537-48.

12. Qi LS, Larson MH, Gilbert LA, Doudna JA, Weissman JS, Arkin AP, Lim WA. Repurposing CRISPR as an RNA-guided platform for sequence-specific control of gene expression. Cell. 2013;152:1173-83.

13. Grossman AD, Erikson JW, Gross CA. The htpR gene product of E. coli is a sigma factor for heat-shock promoters. Cell. 1984;38:383-90.
Ready to submit your research? Choose BMC and benefit from:

- fast, convenient online submission

- thorough peer review by experienced researchers in your field

- rapid publication on acceptance

- support for research data, including large and complex data types

- gold Open Access which fosters wider collaboration and increased citations

- maximum visibility for your research: over 100M website views per year

At BMC, research is always in progress.

Learn more biomedcentral.com/submissions 